\title{
A High-Level Model for Capacitive Coupled RC Oscillators
}

\author{
João Casaleiro and Luís B. Oliveira \\ Dep. Eng. Electrotécnica, Faculdade de Ciência e Tecnologia \\ Universidade Nova de Lisboa, Caparica, Portugal \\ $\{j . c a s a l e i r o, 1 . o l i v e i r a\}$ afct.unl.pt
}

\begin{abstract}
In this paper a high-level model is proposed for quadrature oscillators that are based on two RC oscillators with capacitive coupling. The capacitive coupling technique has the advantages of being noiseless and not increasing the power requirements, which are important features for low power receivers. This coupling technique was already study for LC oscillators but not for RC oscillators. Simulations showed that the behavior of capacitive coupling in RC oscillators is different than for LC. In opposition to what was expected, in RC oscillators, the oscillation frequency increases almost linearly and proportional to the coupling capacitances. This behavior can be explained by the presented model. Simulations are presented to validate the model in respect to the oscillation frequency and to the output phases of the oscillator.
\end{abstract}

Keywords: Quadrature oscillators, RC oscillators, Capacitive coupling, Highlevel model.

\section{Introduction}

Quadrature oscillators are key blocks in the design of modern transceivers. In recent years, significant research efforts have been invested in the study of oscillators with accurate quadrature outputs, with particular emphasis on cross-coupled RC and LC oscillators [1],[2],[3].

Typically, coupled oscillators require two extra gain blocks with current sources. This increases the power dissipation, reduces the oscillation frequency [1], and prevents use of low supply voltages. Recently, capacitive coupling has been proposed for LC oscillators [4],[5] to obtain in-phase and quadrature outputs. This solution, in opposite to the traditional active coupling, does not add extra noise sources and does not increase the power consumption but has the disadvantage of lowering the oscillation frequency.

It is known that inductorless (RC) oscillators [1],[2] occupy less area than LC oscillators. Since they do not require technologies with many metal layers and a thick top layer for the design of high $\mathrm{Q}$ inductors, their fabrication cost is much lower. 


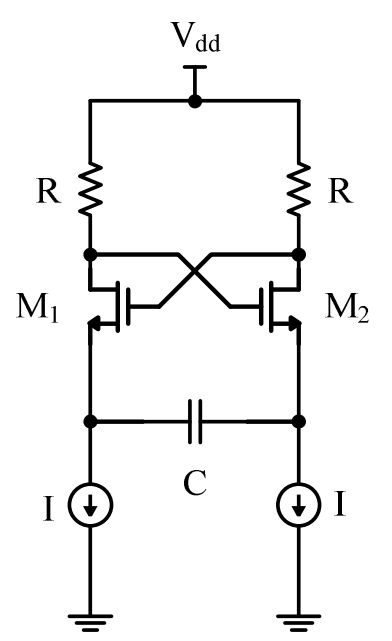

(a)

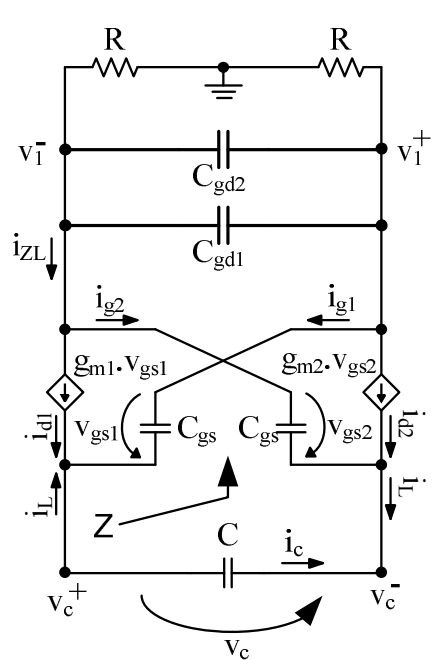

(b)

Fig. 1. a) Single RC oscillator. b) Small-signal circuit

In this paper we investigate quadrature RC oscillators with capacitive coupling and present a novel approach to explain the relation between the coupling factor and the oscillation frequency. Section 2 presents the main contribution of this work for technological innovation. In Section 3, we review the analysis of a single RC oscillator working in the near sinusoidal regime presenting the equations for the amplitude and frequency of the oscillator. In Section 4, the capacitive coupling and the oscillation frequency are discussed and the frequency locking between the two oscillators is explained. The high-level model based on the frequency locking is explained in Section 5. The simulation results are presented in Section 6 and in Section 7, some general conclusions are drawn.

\section{Contribution to Value Creation}

The main application of this work is to aid in the design of RC quadrature oscillators with capacitive coupling, focusing mainly on the low power and low area. With the proposed model the integrated circuit (IC) design time is decreased and the circuit area and power requirements are minimized, which reduce the overall cost of the IC leading to a strong contribution to the value creation.

\section{Single Oscillator}

The source-coupled oscillator, presented in Fig. 1a, working in near sinusoidal regime was extensively study in [6],[7]. 
To determine the oscillation frequency at nearly sinusoidal regime, one needs to determine the impedance, $Z=v_{c} / i_{L}$, seen from the transistors sources, using the small-signal equivalent circuit, without the timing capacitor $C$, as shown in Fig. $1 \mathrm{~b}$. Assuming that the circuit has no mismatch, the impedance, $Z$, is given by,

$$
Z(s) \approx \underbrace{2\left(\frac{1}{g_{m}}-R\right)}_{\text {Real term- } R_{e}}+\underbrace{s 2 \frac{R}{g_{m}}\left(4 C_{g d}+C_{g s}\right)}_{\text {Imaginary term- } j \omega L_{e}}
$$

Where $g_{m}$ represents the transistor $M_{1}$ and $M_{2}$ transconductances, $C_{g d}$ and $C_{g s}$ are the gate to drain capacitances and gate to source capacitances respectively of both transistors $M_{1}$ and $M_{2}$.

From (1) one can conclude that the circuit can be simplified to a RL circuit. And that the overall circuit is an RLC. In order to oscillate, the real part of (1), $R_{e}$, must be zero or negative, which is rather simple if the resistors value is higher than the inverse of $M_{1}$ and $M_{2}$ transconductances, i.e. $R>1 / g_{m}$. In this case, the oscillation frequency is given by

$$
\omega=\frac{1}{\sqrt{2 \frac{R}{g_{m}}\left(4 C_{g d}+C_{g S}\right) C}}
$$

Where $C$ is the timing capacitor of the oscillator.

To determine the steady-state amplitude is necessary to add the nonlinearities of the circuit, which will limit the amplitude of oscillation and introduce a neglectable shift on the oscillation frequency if timing capacitor $C$ is much larger than the sum of gate to drain capacitances $C_{g d}$. The strongest nonlinearities of the circuit are introduced by the transconductances of transistor $M_{1}$ and $M_{2}$, which suffer a drift from their nominal value for large signal swings. Thus, to determine the oscillator differential equation one must assume that the transconductance $g_{m l}$ and $g_{m 2}$ are different, $g_{m l} \neq g_{m 2}$. The oscillator characteristic equation can be reduced to the Van der Pol equation [5], using the notation $x=i_{L} / I$,

$$
\frac{d^{2} x}{d t^{2}}-2\left(\delta_{0}-\delta_{2} x^{2}\right) \frac{d x}{d t}+\omega_{0}^{2} x=0
$$

where

$$
\delta_{0}=\frac{R g_{m 0}-1}{2 R\left(4 C_{g d}+C_{g s}\right)}
$$

and

$$
\delta_{2}=\frac{R g_{m 0} K}{2 R\left(4 C_{g d}+C_{g s}\right)}
$$

where $K$ is a fitting parameter to compensate the equations simplification.

The solution for (3) is of the form, 


$$
x(t)=A \cdot \sin \left(\omega_{0} t\right)
$$

where $A$ represents the amplitude of oscillation that is given by

$$
A \approx 2 I \sqrt{\frac{R g_{m 0}-1}{K R g_{m 0}}}
$$

where $I$ is the circuit bias current.

\section{$4 \quad$ Frequency Locking}

When a differential current is injected in parallel with the timing capacitor the oscillator will behave like a phase lock loop, as shown in the simulation results section. The oscillator will lock to the frequency of the injected current and the instantaneous oscillation frequency can be approximated by

$$
f \approx f_{0}+\frac{i_{e}}{i_{c}} \cdot \cos (\Delta)
$$

where $f_{o}$ represents the nominal oscillation frequency without injected current, $i_{e}$ is the injected current, $i_{c}$ is the current in the timing capacitor, and is the phase difference between $i_{e}$ and $i_{c}$. The capture and locking range depends on the $i_{e} / i_{c}$ ratio.

When two RC oscillators are coupled using capacitors, as shown in Fig 2, both oscillators lock to each other thought the currents $i_{e 12}$ and $i_{e 21}$. The current $i_{e 12}$ represents the injected current of oscillator one into oscillator two and $i_{e 2 l}$ represents the opposite. These injected currents will force both oscillators to adjust their frequency, amplitudes and phases.



Fig. 2. Circuit of a quadrature oscillator based on two capacitive coupled RC oscillators

Assuming no mismatch leads to equal amplitudes and the same free running frequency $f_{0}$ for both oscillators. 


\section{$5 \quad$ High-Level Model}

The high-level model for the capacitive coupled RC oscillators is presented on Fig. 3.

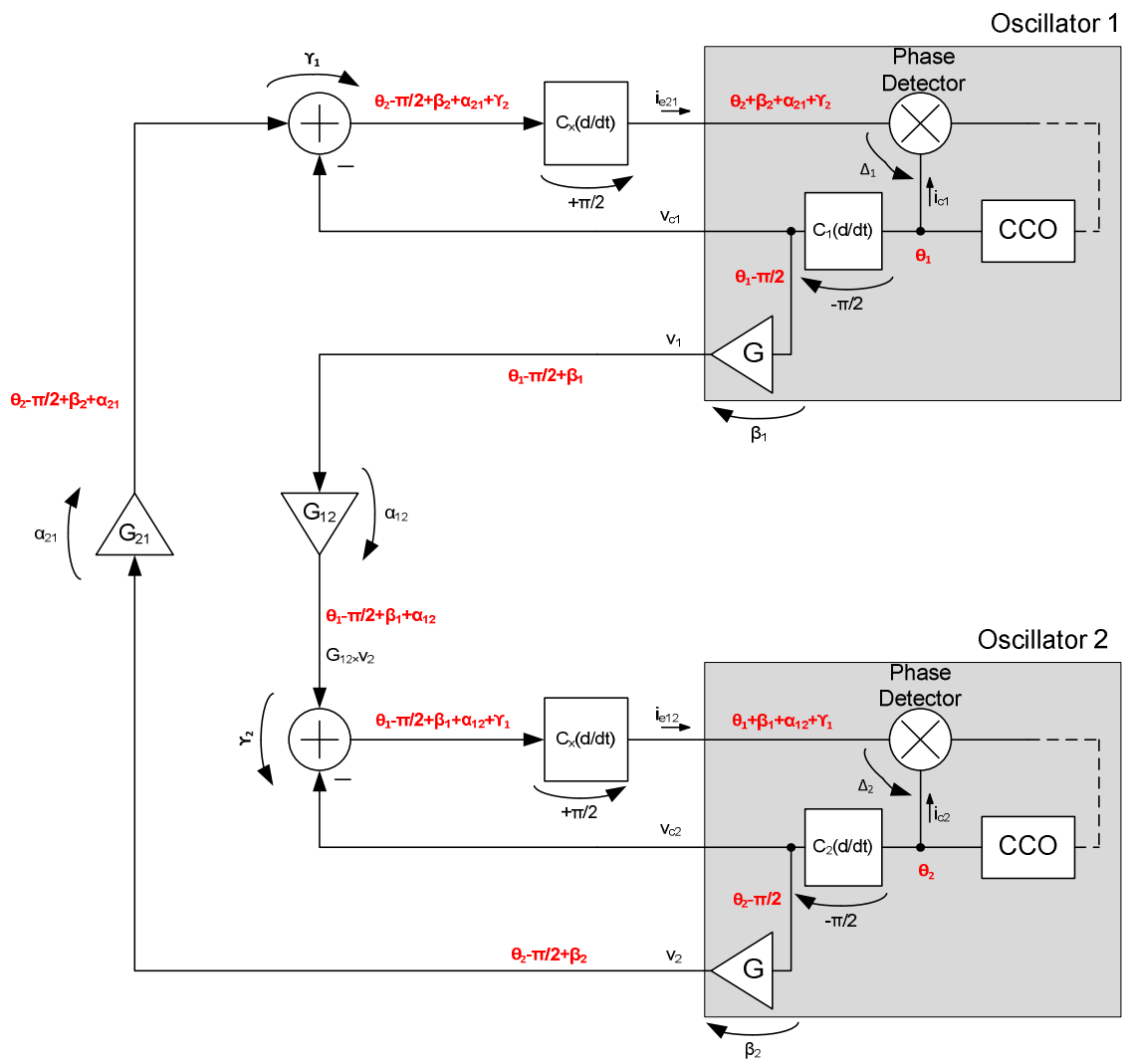

Fig. 3. The block diagram of the high-level model with the phase shift of each block

Where the oscillators are represented by a first-order phase lock loop (PLL) - gray box -, with the instantaneous frequency (8). The coupling is modeled by two gain blocks, $G_{21}$ and $G_{12}$; and the conversion from voltage to current - performed by the coupling capacitors $C_{X}$ - are modeled by differential blocks with gain $C_{X}$.

Assuming a locked state, where the frequency of oscillation is equal for both oscillators, and assign generic phase shifts for each block, one can derive the following system of equations:

$$
\left\{\begin{array}{l}
\Delta_{1}=\left(\theta_{2}+\beta_{2}+\alpha_{21}+\gamma_{2}\right)-\theta_{1} \\
\Delta_{2}=\left(\theta_{1}+\beta_{1}+\alpha_{12}+\gamma_{1}\right)-\theta_{2}
\end{array}\right.
$$

where $\Delta$ represents the phase difference between currents $i_{e}$ and $i_{c}, \theta$ is the oscillator absolute phase, $\beta$ represents the phase shift between lower and upper parts 
of the oscillator, i.e. phase between $v_{c}$ and $v, \alpha$ is the phase shift introduced by the coupling and $r$ the phase difference inserted by the amplitude difference in the coupling capacitor. All symbols are indexed accordingly to its oscillator number.

Assuming no mismatch $\Delta_{1}=\Delta_{2}, \beta_{1}=\beta_{2}$ and $\gamma_{1}=\gamma_{2}$, the phase difference between the oscillators are given by

$$
\left(\theta_{2}-\theta_{1}\right)=\frac{\alpha_{12}-\alpha_{21}}{2}
$$

From (10) it is possible derive the values in Table I, which are in accordance with the conclusions of [8], that a system with $N$ coupled differential oscillators produces outputs with a phase shift of $\pi / N$. To obtain quadrature outputs one of the couplings should be direct and other crossed.

Table 1. Oscillator phase output

\begin{tabular}{|c|c|c|c|c|c|}
\hline \multicolumn{2}{|c|}{$\begin{array}{c}\text { Coupling } \\
\text { Oscillator } 1 \text { to Oscillator2 }\end{array}$} & \multicolumn{2}{|c|}{$\begin{array}{c}\text { Coupling } \\
\text { Oscillator } 2 \text { to Oscillator1 } \\
\end{array}$} & \multicolumn{2}{|c|}{$\begin{array}{l}\text { Phase } \\
\text { output }\end{array}$} \\
\hline$G_{12}$ & $\alpha_{12}$ & $G_{21}$ & $\alpha_{21}$ & \multicolumn{2}{|c|}{$\theta_{2}-\theta_{1}$} \\
\hline 1 & $0 \quad(2 \pi)$ & 1 & $0 \quad(0)$ & $0(\pi)$ & In-phase \\
\hline-1 & $\pi$ & 1 & 0 & $\pi / 2$ & Quadrature \\
\hline 1 & 0 & -1 & $\pi$ & $\pi / 2$ & Quadrature \\
\hline-1 & $\pi \quad(2 \pi)$ & -1 & $\pi \quad(0)$ & $0(\pi)$ & In-phase \\
\hline
\end{tabular}

If both couplings are either crossed or direct then outputs can be in-phase or in opposition of phase (dependent on the initial conditions).

The dependence of the frequency by the coupling capacitances $C_{X}$ can be explained using (8). If one increased the value of $C_{X}$ the current $i_{e}$ also increase and as a result the frequency will be dependent on the term $\cos ()$. If the cosine value is positive which is the case for $\theta_{2}-\theta_{l=} \pi / 2$ - the frequency increases. If the cosine value is negative - which is the case for $\theta_{2}-\theta_{1=} 0$ - the frequency decreases.

\section{Simulations and Results}

The circuit in Fig. 2 was simulated using the Cadence Spectre and SpectreRF and UMC $130 \mathrm{~nm}$ CMOS technology library. The oscillator was designed for a nominal frequency, $f_{0}$, around $1.9 \mathrm{GHz}$. Transistors $M_{1}, M_{2}, M_{3}$ and $M_{4}$ have $\mathrm{W}=7.2 \mu \mathrm{m}, \mathrm{L}=$ $120 \mathrm{~nm}$. The current sources $I$, implemented as single transistors with $\mathrm{W}=7.2 \mu \mathrm{m}, \mathrm{L}=$ $360 \mathrm{~nm}$ (not shown in the circuits). The resistors (Fig. 2) are also from the technology library; they have $\mathrm{W}=1 \mu \mathrm{m}$ and $\mathrm{L}=1.6 \mu \mathrm{m}$ which results in $R=185.4$. The timing capacitance are implemented using two parallel capacitors with $\mathrm{W}=11 \mu \mathrm{m}$ and $\mathrm{L}=$ $22 \mu \mathrm{m}$, resulting in a capacitance of $431.7 \mathrm{fF}$. The coupling capacitances $C_{x}$ was changed from $0 \mathrm{fF}$ up to $200 \mathrm{fF}$. The supply voltage is $1.2 \mathrm{~V}$, and the bias current is $550 \mu \mathrm{A}$. 
Fig. 4 shows the results of the simulations - for several frequencies - of a single $\mathrm{RC}$ oscillator with a current source $i_{e}$ in parallel with the timing capacitor. As can be seen the oscillator locks to the frequency of the external source - up to the limit imposed by the $i_{e} / i_{c}$ ratio - and the phase difference between both currents is presented in Fig. 4b. The oscillator returns to its nominal frequency $f_{0}$ when the external/forced frequency is above the locking range. This result clear shows that the oscillator behaves like a phase lock loop.

Fig. 5 shows the simulation results of two coupled RC oscillators (circuit of Fig. 2). The value of the coupling capacitances was changed from 0 to $200 \mathrm{fF}$. Fig. $5 \mathrm{~b}$ shows the frequency increase almost linear in relation to the $C_{X}$ value. Fig. 5a shows that a coupling capacitance above $10 \mathrm{fF}$ lock the two oscillators to each other and generate four phases with $\pi / 2$ phase difference.

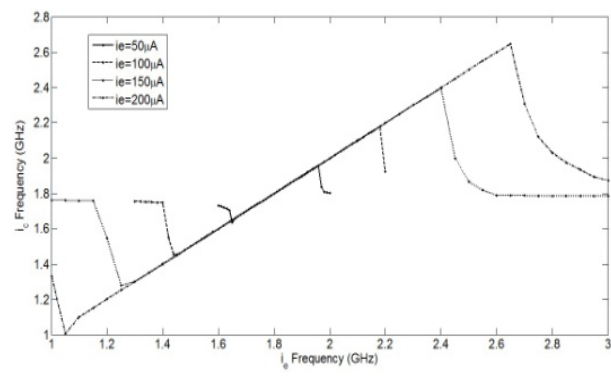

(a)



(b)

Fig. 4. a) Frequency of $i_{e}$ and $i_{c}$; b) The $i_{c}$ and $i_{e}$ phase difference

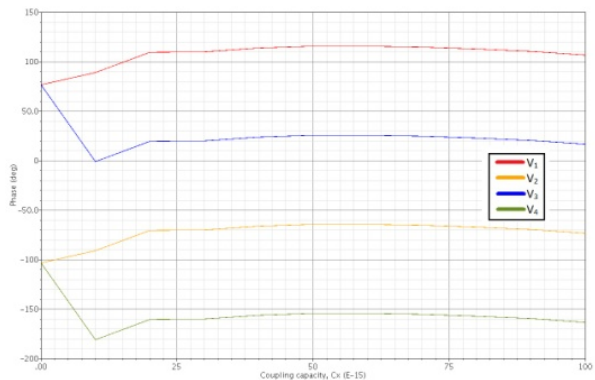

(a)

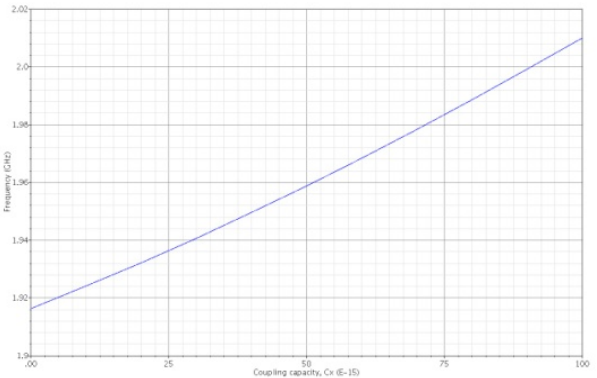

(b)

Fig. 5. Phase difference between $i_{e}$ and $i_{c}$ as a function of the $i_{e}$ frequency

\section{Conclusions}

This work presented a high-level model for RC quadrature oscillators capacitive coupled and demonstrated by simulations that the RC oscillator behaves like a PLL. The objective of the model is to better explain the three possible modes of oscillation 
- in-phase, quadrature and phase opposition - and the frequency relation to the coupling factor for all modes.

Simulations showed that the in-phase and phase opposition modes are strongly dependent of the circuit initial conditions. Essential because for non-crossed and double crossed connections both modes are stable. In these two modes the frequency always decrease when the coupling factor grow. For the quadrature mode the frequency increases proportional to the coupling factor.

The locking range of each oscillator is directly proportional to the ratio of the coupling currents and the timing capacitor current.

\section{References}

1. Oliveira, L.B., Fernandes, J.R., Filanovsky, I.M., Verhoeven, C.J.M., Silva, M.M.: Analysis and Design of Quadrature Oscillators. Springer, Heidelberg (2008)

2. Razavi, B.: RF Microelectronics. Prentice-Hall (1998)

3. Fernandes, J.R., Kouwenhoven, M.H.L., van den Bos, C., Oliveira, L.B., Verhoeven, C.J.M.: The Effect of Mismatches and Delay on the Quadrature Error of a Cross-Coupled Relaxation Oscillator. IEEE Transactions on Circuits and Systems I: Regular Papers 54(12), 2592-2598 (2007)

4. Fu, C.T., Luong, H.C.: A 0.8-V CMOS quadrature LC VCO using capacitive coupling. In: IEEE Asian Solid-State Circuits Conference, ASSCC 2007, San Francisco, pp. 436-439 (2007)

5. Filanovsky, I.M., Verhoeven, C.J.M.: Sinusoidal and Relaxation Oscillations in SourceCoupled Multivibrators. IEEE Transactions on Circuits and Systems II: Express Briefs 54(11), 1009-1013 (2007)

6. Buonomo, A., Lo Schiavo, A.: Analysis of emitter (source)-coupled multivibrators. IEEE Transactions on Circuits and Systems I: Regular Papers 53(6), 1193-1202 (2006)

7. Filanovsky, I.M., Verhoeven, C.J.M.: Sinusoidal and relaxation oscillations in emittercoupled multivibrators. In: 50th Midwest Symposium on Circuits and Systems, MWSCAS 2007, Montreal, Canada, pp. 469-472 (2007)

8. Romano, L., Levantino, S., Samori, C., Lacaita, A.L.: Multiphase LC Oscillators. IEEE Transactions on Circuits and Systems I: Regular Papers 57(7), 1579-1588 (2006)

9. Jia, L., Ma, J.-G., Yeo, K.S., Do, M.A.: 9.3 - 10.4 GHz Band Cross-Coupled Complementary Oscillator with Low Phase-Noise Performance. IEEE Transactions on Microwave Theory and Techniques 52(4), 1273-1278 (2004)

10. van der Tang, J., Kasperkovitz, D., van Roermund, A.: A 9.8-11.5-GHz quadrature ring oscillator for optical receivers. IEEE Journal of Solid-State Circuits 37(3), 438-442 (2002)

11. Oliveira, L.B., Filanovsky, I.M., Allam, A., Fernandes, J.R.: Synchronization of two LCoscillators using capacitive coupling. In: IEEE International Symposium on Circuits and Systems, ISCAS 2008, Seattle, pp. 2322-2325 (2008) 